\title{
Un homme est un homme
}

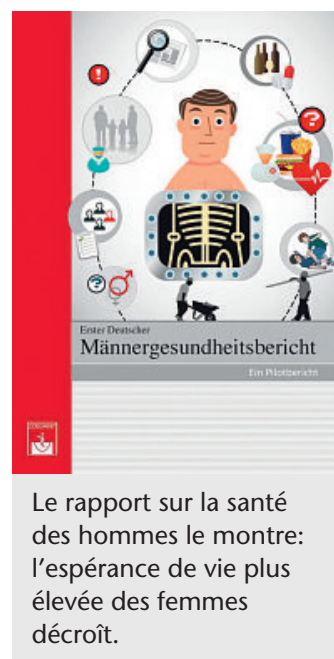

1 Bardehle D, Stiehler M (ed.). Erster Deutscher Männergesundheitsbericht. München: Zuckschwerdt. 2010.
Les temps héroïques où les hommes combattaient des dragons ou cédaient les canots de sauvetage du Titanic aux femmes et aux enfants sont révolus. L'image du chevalier a complètement changé dans notre société de prestations de services. Assurer sa masculinité est manifestement devenu plus difficile à tous les âges. Le sexe dit fort souffre de crises d'orientation, avale de la Ritaline, mute en casseur quand il est jeune et vote plus tard pour la droite conservatrice, se bat pour le droit de garde parental et fuit dans une maison des pères lorsque les femmes et la gauche prennent à nouveau le pouvoir. C'est en tous les cas l'impression donnée par la presse quotidienne et les nouvelles radiodiffusées ou télévisées.

La santé virile en prend un coup depuis quelque temps déjà: sperme de mauvaise qualité, produits ramollissants et pesticides dans l'urine ou cadavres imbibés de vodka dans l'ancienne Union soviétique. L'espérance de vie différente entre les sexes est un sujet de recherche depuis quelques décennies. «Une maladie dénommée homme» titrait, il a quelques années le Spiegel, l'homme, un «idiot de la santé résistant aux conseils» et un «cas de santé à assainir», soulignent d'autres auteurs. Longuement attendu, le «premier rapport sur la santé en Allemagne»publié en 2010 contient enfin des données claires et précises [1]. Des experts d'horizons variés y expliquent les déficits de la santé masculine et les mesures à prendre. Ce rapport de 198 pages a vu le jour grâce à la «Fondation pour la santé masculine» et la «Société allemande pour l'homme et sa santé», avec le soutien de $l^{\prime}$ «Assurance-maladie allemande». Les débats portent sur la répartition du travail et l'image traditionnelle de l'homme. Les vertus soldatesques ne servent plus de modèle pour réussir, et le comportement dommageable dû à l'héritage historique nécessite encore beaucoup d'éducation. En Allemagne, les femmes vivent 5 ans $1 / 3$ plus longtemps; elles n'atteignent une espérance de vie plus élevée qu'en Norvège, aux PaysBas, en Italie, en France, en Suède, en Suisse, en Espagne et au Royaume-Uni.

La première mesure recommandée par les professionnels de la santé consiste à faire baisser la mortalité précoce due au cancer du poumon, aux maladies cardio-vasculaires, à l'hypertonie, aux maladies du foie, aux dépressions, à la violence, aux accidents et au suicide. Les risques et les problèmes font l'objet d'une statistique analysée et commentée pour chaque groupe d'âge. Entre-temps, la recherche effectuée dans les couvents a montré qu'à des conditions de vie très semblables, l'espérance de vie des moines et des nonnes était presque identique. L'avantage génétique des femmes ne leur accorde qu'une année de plus et l'écart le plus grand est dû à des facteurs culturels et sociaux, par exemple, aux conditions de travail, à l'individualisation économique forcée, aux contraintes de la mondialisation, au chômage, aux causes migratoires et à l'aveuglement de la médecine en la matière. «C'est le développement des inhibiteurs PDE5 mais surtout les statistiques sur la santé qui ont révélé que les hommes avaient aussi des problèmes spécifiques à prendre en considération.» Les problèmes masculins restent trop dans l'ombre, on manque de données fiables, de recherche et d'offres préventives et thérapeutiques. La Fondation suisse pour la santé RADIX constatait déjà en 2005 qu'en comparaison avec les activités menées en faveur des femmes, la recherche médicale et la pratique axées spécifiquement sur la santé masculine restaient une exception en Suisse. Mais il est aussi important de ne pas mettre en concurrence les intérêts des hommes et des femmes en matière de santé. La diversité masculine et la diversité féminine doivent avoir les mêmes chances dans leur vécu au quotidien.

L'Office fédéral de la statistique a analysé la mortalité des personnes nées entre 1900 et 2008 et a développé un modèle mathématique jusqu'en 2030 sur cette base. Tous les trois ans, la durée de vie moyenne a augmenté d'un an. Les femmes détiennent les records, néanmoins leur avance est marquée par une tendance à la baisse au cours des générations. Les hommes rattrapent peu à peu leur retard de sorte que leur gain en espérance de vie dépasse celui des femmes pour les années de naissance après 1940. «Vive la différence», peut-être n'est-elle ici aussi pas tant grande, comme le suggère un marché de produits médico-pharmaceutiques lucratif, axé sur l'esthétique. Un homme est un homme, en particulier dans notre pays où la présence d'un fusil dans l'armoire à balais renforce l'identité chancelante du père de famille en état de se défendre, malgré les mouvements de protestation masculine et les démonstrations anti-féministes: le patriarcat reste bien en selle. Les mutations de notre société sont un fait, mais elles se produisent de façon bien plus complexe que ne le montrent les rapports statistiques sur la santé des hommes et des femmes. Néanmoins, il ne faut pas sous-estimer les répercussions de ces études, en particulier lorsque les médecins les prennent au sérieux.

Erhard Taverna 